\title{
Characteristics of Tuberculosis among Children and Adolescents at a Referral TB’s Hospital, 2006 - 2011
}

\author{
Mohammad Reza Bolursaz, Ferial Lotfian,, ${ }^{1}$ Farahnaz Aghahosseini,, ${ }^{2}$ Maryam Hassanzad, ${ }^{1}$ Hosseinali \\ Ghafaripoor, ${ }^{3}$ Soheila Khalilzadeh, ${ }^{1}$ Noshin Baghaie, ${ }^{1}$ and Aliakbar Velayati ${ }^{2}$ \\ ${ }^{1}$ Pediatric Respiratory Diseases Research Center, National Research Institute of Tuberculosis and Lung Diseases (NRITLD), Masih Daneshvari Hospital, Shahid Beheshti \\ University of Medical Sciences, Tehran, IR Iran \\ ${ }^{2}$ Clinical Tuberculosis and Epidemiology Research Center, National Research Institute of Tuberculosis and Lung Diseases (NRITLD), Masih Daneshvari Hospital, Shahid \\ Beheshti University of Medical Sciences, Tehran, IR Iran \\ ${ }^{3}$ Chronic Respiratory Diseases Research Center, National Research Institute of Tuberculosis and Lung Diseases (NRITLD), Shahid Beheshti University of Medical Sciences, \\ Tehran, IR Iran \\ "Corresponding author: Ferial Lotfian, Pediatric Respiratory Disease Research Center, Masih Daneshvari Hospital, Shahid Beheshti University of Medical Sciences, Tehran, IR \\ Iran. Tel: +98-2127122003, Fax: +98-2120109484, E-mail: lotfianferial@yahoo.com
}

Received 2016 June 18; Accepted 2016 June 23.

\begin{abstract}
Background: Tuberculosis (TB) is a globally significant cause of morbidity and mortality in children. Few data on tuberculosis in children and adolescents are available in Iran.

Objectives: The current study aimed to describe the case characteristics and clinical-epidemiological aspects of children and adolescents with TB.

Methods: A retrospective review was undertaken on 203 patients aged less than 19 years admitted to a referral TB hospital from 2006 to 2011.

Results: Out of the 203 children and adolescents diagnosed with TB, 57.6\% of cases were female. Median age was 15 years and $51 \%$ were 10 -18 years old; $83 \%$ had pure pulmonary TB. The common type of extrapulmonary TB (EPTB) was pleuritis (64\%) and $80 \%$ of the EPTB cases were observed in adolescents aged $15-18$ years. Female adolescents aged 15-18 years were more likely to have positive smear (88\%), cultural growth (63.6\%) and drug resistant TB infection (71\%).

Conclusions: In this referral hospital setting, more pediatric patients with TB were found among adolescent cases especially females. Early detection of adolescents at risk to developing infection is the essential constituent of TB control.
\end{abstract}

Keywords: Children, Adolescents, Tuberculosis, Female, Extra Pulmonary

\section{Background}

Tuberculosis (TB) is a globally significant cause of morbidity and mortality in children. The world health organization (WHO) estimated the global burden of tuberculosis at 9.6 million new cases in 2014 (1). In 2015, the WHO estimated that 1 million children develop TB worldwide whom more than 136,000 die each year (2). The epidemiology of pediatric TB depends on risk factors such as race, age, immigration, overcrowding, poverty and HIV (3). Few data are published on the epidemiology of pediatric TB in the new millennium and WHO asked for more researches in this field especially in the developing countries (4). Pediatric TB is a challenging disease in diagnosis and therapeutic options. The difficulty of achieving sufficient amount of specimens and low rate of bacteriologic confirmation are well known, particularly in infants and young children (5). Although the role of pediatric TB in the transmission of dis- ease may be lower than that of adult patients, pediatric TB can be a reservoir which constitutes a significant number of future adult cases (6). Pediatric TB usually suggests current transmission, most usually from an infectious adult with cavitary disease or pulmonary TB. Children are considerably at high risk of progression to severe forms of the disease such as TB meningitis and miliary TB (7). To improve early detection and diagnosis of tuberculosis in children, it is recommended to perform routine screening of children in close contact with infectious adult patients (8). Few data on children and adolescents with tuberculosis in Iran are available for physicians to manage such patients.

\section{Objectives}

Understanding the clinical presentations of tuberculosis in pediatrics is essential for early diagnosis and disease 
control. Therefore, the current study aimed to determine clinical features of TB in children and adolescents.

\section{Methods}

From March 2006 to March 2011, 203 patients less than 18 years old with confirmed diagnosis of TB were admitted to TB wards of National research institute of tuberculosis and lung disease (NRITLD) in Masih Daneshvari Medical Center, Tehran, Iran. These patients were reviewed retrospectively. Patients were categorized by age into four groups: less than 5 years, 5 - 9 years, 10 - 14 years and 15 18 years. The following data were analyzed for the current study: demographic data, presenting symptoms, clinical features, bacteriological results, tuberculin skin test (TST) and drug susceptibility results. TB was defined as the attendance of chronic symptoms (fever, chronic cough, weight loss or failure to thrive) based on the radiological manifestations (hilar lymphadenopathy and/or consolidation) and the histological appearance of biopsy material similar to the appearance of TB-affected tissues (caseous necrosis or granulomatous tubercles); or the separation of Mycobacterium tuberculosis (M. tuberculosis) from sputum, gastric lavage, body secretions and surgical specimens. In this retrospective study, institutional review board approval was not required.

\section{Results}

A total of 203 patients were enrolled into the study. TB was diagnosed in 115 cases in the first three years of the study (2006 - 2008) and 88 cases in the second three years (2009-2011). Baseline characteristics are presented in Table 1. The median age of the patients at the time of TB diagnosis was 15 years (ranging from four months to eighteen years). Twenty-eight (13.8\%) cases were identified by contact investigation. About $56 \%$ of the patients had an adult case of TB in their families.

Co-morbidities were identified only in six patients which two of them had HIV co-infection; and one had HIV and HBV co-infection. Distribution of TB site according to the age is described in Table 2.

The most frequent sites of extrapulmonary TB were pleural $(n=23)$, central nervous system $(\mathrm{CNS})(\mathrm{n}=5)$, lymph nodes $(\mathrm{n}=3)$, bone and joints $(\mathrm{n}=3)$, and miliary TB $(\mathrm{n}=1)$, as well as peritonitis $(\mathrm{n}=1)$ (Table 3$)$.

In 102 (50.3\%) patients, TB was confirmed by the isolation of M. tuberculosis including sputum in 66 cases (64.7\%), gastric aspirates $20(19.6 \%)$, sputum and gastric aspirates 10 (9.8\%), bronchus 4 (3.9\%), gastric aspirates, sputum and pleural effusion $1(1 \%)$, as well as sputum and lymph-node biopsy 1 case (1\%). Sixty (90.9\%) patients with positive sputum smear aged 15 - 18 years which 53 (88\%) of them were female.

Cultures were performed for all samples. Positive culture for M. tuberculosis were detected in $33(16.3 \%)$ patients while $21(63.6 \%)$ of them were female. Age group for 20 (60.6\%) of culture positive cases was 15 -18 years old. Among patients with positive culture, 31(93.9\%) cases were the positive acid fast bacilli (AFB) smears. Twenty-two patients were confirmed as TB cases by a histological diagnosis (pleural $\mathrm{n}=12$, bronchial $\mathrm{n}=5$, lymph nodes $\mathrm{n}=3$, bone and joint biopsy $n=2$ ). Among 203 patients diagnosed as TB cases, 136 underwent TST which 74 cases out of them were TST positive. Sixty-nine of the 74 (93.2\%) cases had indurations $\geq 10 \mathrm{~mm}$ tuberculin testing.

Twenty-one patients (10.34\%) did not respond to standard anti-TB, which drug susceptible test was registered for seven patients; all except ones were 10 - 18 years old and only one patient was a three-year-old Afghan girl. Fifteen (71.4\%) patients unresponsive to anti-TB were female. Resistance to isoniazid, isoniazid-rifampin, isoniazid-rifampinetambotol and streptomycin was found in 2, 3, 1 and 1 strains, respectively.

\section{Discussion}

Tuberculosis was a main health problem in Iran in the past; however, the burden of disease decreased in the last four to five decades (9). In 2010, WHO reported a $10 \%$ decline in TB incidence rate in Iran (10). It suggests good improvement in case detection rate at the national level. However, according to 2012 WHO global report, Iran was reported a member of 104 countries that contain $94 \%$ of the world's TB cases (2); therefore, studying the epidemiology of tuberculosis is still important. The continuous attention to prevent TB among children and adolescents is critical to sustain these decreases and achieve the breakthrough improvement of a TB free generation. In the current six-year study, TB cases decreased in the second threeyear period (2009 - 2011) compared to the first three-year period (2006 - 2009). The most prevalent age for infection was the adolescent period (15-18 years) and the second peak occurred in the 10 - 14 years; in these age groups, female patients were more than male ones. Ghadiri et al. reported the similar age distribution of TB in two age groups in Kermanshah, Iran (11). Sharifi-Mood et al. found that the prevalence of TB increased by increasing age and the population of female patients also rose in Zahedan, Iran (12). The recent studies showed that adolescents are a vulnerable age group with a higher chance of developing pulmonary TB disease compared to younger children $(13,14)$. A previous study among 2185 patients of 0 - 14 years in Iran 
Table 1. Baseline Characteristics of Children and Adolescents With Active Tuberculosis ${ }^{\mathrm{a}}$

\begin{tabular}{|c|c|}
\hline Characteristic & Patients \\
\hline \multicolumn{2}{|l|}{ Age, $y$} \\
\hline$<5$ & $26(12.8)$ \\
\hline $5-9$ & $29(14.3)$ \\
\hline $10-14$ & $44(21.7)$ \\
\hline $15-18$ & $104(51.2)$ \\
\hline \multicolumn{2}{|l|}{ Gender } \\
\hline Male & $86(42.4)$ \\
\hline Female & $117(57.6)$ \\
\hline \multicolumn{2}{|l|}{ Race } \\
\hline Iranian & $105(51.7)$ \\
\hline Afghan & $98(48.3)$ \\
\hline Underlying medical condition & $6(3)$ \\
\hline
\end{tabular}

${ }^{\mathrm{a}}$ Values are expressed as No. (\%).

reported that most of the patients were less than five years old (15). The difference, thus, may be due to the fact that small size of the current study population and TB disease is likely the result of reactivation in lately infected children rather than current transmission in the community. Consequently, population-based studies are required to determine the true incidence of tuberculosis disease in children and adolescents in Iran.

In the current study, adolescents had more positive smear and culture than children. The pulmonary tuberculosis disease is frequently characterized by pulmonary parenchymal cavitation throughout puberty (16). Since this age group has extensive social combination and assembly in schools and colleges, these patients are able to transmit the ТВ infection to others. Furthermore, the adolescent age group is specific for physiological changes which occur during puberty and play an important role in pulmonary TB disease (17). This can illustrate the higher risk to get TB after recent TB infection and re-infection among adolescent age group compared with children. The management of the TB in adolescent patients will decrease the prevalence of TB infection.

The current study also documented a trend toward higher prevalence of extrapulmonary TB disease in adolescents $(10.4 \%)$ than in young children (3.8\%), the group was previously determined as having the highest prevalence of extrapulmonary TB disease. The studies in Canada and France confirmed the high prevalence of extrapulmonary disease in adolescents $(13,18,19)$. In another study in Zahedan, Iran, patients aged 13 - 18 years old had more extrapulmonary type of TB than the other age groups (12).
Sharifi-Mood et al. (12) identified that among 195 patients with TB aged less than 18 years, $23 \%$ had extrapulmonary TB that TB lymphadenitis and pleuritis were the most common sites of TB. In the current study, TB pleuritis and CNS TB were the common forms. Phongsamart et al. showed that TB lymphadenitis was the most common site in 121 patients less than 17 years in a population-based study in Canada (13). In a recent study in China out of 1212 children aged 0 - 18 years, the most common types of extrapulmonary TB were TB meningitis and disseminated TB which most pediatric patients with EPTB aged less than five years old. They concluded that pediatric cases with the history of close contact with TB infected patients and no Bacillus Calmette-Guerin (BCG) vaccination might have a higher risk of severe TB (20). Thus, BCG vaccination at birth and better detection of TB-infected children with contact to adult patients with TB can help to prevent extrapulmonary and severe TB in young children (21). High prevalence of EPTB in adolescents in the current study may be due to previously developed latent TB infection which some factors can lead to disease in this group.

In the current study, about $60 \%$ of patients aged $10-14$, and 15 - 18 years were female. Several studies in different countries show that the progression to disease and mortality rates are higher in females during reproductive years, while males have higher rate during the following years in life $(22,23)$. Currently, evidence suggests a role for steroid hormone in immune response $(24,25)$. Few studies show the role of steroids hormone in susceptibility or protection of human to infectious diseases. Therefore, investigations should be conducted to identify the effect of sexual hor- 
Table 2. Symptoms of Children and Adolescents With Active Tuberculosis in the Study

\begin{tabular}{|c|c|}
\hline Complaint & Patients \\
\hline Cough & $150(23.6)$ \\
\hline Fever & $111(17.5)$ \\
\hline Weight loss & $102(16.1)$ \\
\hline Night sweats & $69(10.9)$ \\
\hline Dyspnea/respiratory failure & $56(8.8)$ \\
\hline Chest pain & $36(5.7)$ \\
\hline Hemoptysis & $20(3.1)$ \\
\hline Abdominal pain/distension & $14(2.3)$ \\
\hline Headache/seizure/muscle weakness & $5(0.8)$ \\
\hline Generalized lymphadenopathy & $4(0.6)$ \\
\hline Neck mass & $2(0.3)$ \\
\hline Back pain & $1(0.2)$ \\
\hline
\end{tabular}

${ }^{\mathrm{a}}$ Values are expressed as No. (\%).

Table 3. Number of Patients and Sites of Tuberculosis Diagnosed for Different Age Groups

\begin{tabular}{|c|c|c|c|c|c|c|c|}
\hline Age, $y$ & Pulmonary & Pleural & CNS & Lymphadenitis & Osteoarticular & GI & Miliary \\
\hline$<5$ & 26 & & & & & & \\
\hline $5-9$ & 29 & & & & $1^{a}$ & & \\
\hline $10-14$ & 42 & $2+3^{\mathrm{a}}$ & & & & & \\
\hline $15-18$ & 92 & $10+8^{a}$ & $5^{\mathrm{a}}$ & $1+1^{\mathrm{a}}$ & $1+1^{\mathrm{a}}$ & $1^{\mathrm{a}}$ & 1 \\
\hline
\end{tabular}

${ }^{a}$ Case with concomitant other sites of tuberculosis diagnosed.

mones on susceptibility differences between female and male patients with TB. The nutritional status, an important factor associated with immune function, is suggested to participate in the role of individual response to tuberculosis. The role of vitamin D in the immune response to tuberculosis is identified; macrophages infected with $M y$ cobacterium tuberculosis need 25-hydroxyvitamin D to begin the immune response (26). Several studies showed that female children and adolescents in Iran have low level of 25-hydroxyvitamin D (27-29).

Other micronutrients such as iron play an important role in the immune function. It is revealed that iron deficiency compromises cell-mediated immunity, reducing macrophage activity and declining T-cell numbers $(30,31)$. WHO estimates that $39 \%$ of children younger than five years, $48 \%$ of children $5-14$ years, $52 \%$ of pregnant females, and $42 \%$ of all females in the developing countries are anemic whereas half of which have iron deficiency(32). Recent studies indicated that iron deficiency has an important role in disease progression and poor clinical outcomes (33, 34). Strategies to preserve normal iron status in TB cases could be useful to decrease TB morbidity and mortality. In the current study, the smear-positive pediatric TB was found more in females 15 - 18 years than male TB cases of the same age. It is confirmed that adolescent females have a tendency to develop adult-type sputum smear-positive TB more than males (34).

The drug resistant TB cases are significantly increasing. WHO reports that the cases of MDR-TB are increasing in the world (35). However, few data are available on the incidence and prevalence of MDR-TB in children. The lack of data on drug-resistant TB among children may be due to the fact that drug susceptibility testing (DST) and cultures for $M$. tuberculosis are not regularly performed, since it is not simple to get enough specimens in children TB suspects and mycobacterium culture yields are low (35). The novel strategies that specially address pediatric TB diagnosis and management should be considered. Pediatric 
TB services require improving diagnostic ability including newer diagnostic tools and progression of the current diagnostic tools. Compromise diagnostic algorithms are needed in children and adolescent cases with TB such as recently algorithm planned by Graham et al. (36) to improve the standard of care for pediatric patients with TB. Kaufmann et al. provided new vaccines and long-term vaccination schedules to target the goal of decreasing the yearly incidence of TB to less than one new case per million by 2050 (37). The Xpert® MTB/RIF assay is a new molecular diagnostic tool established to be more accurate than microscopy to detect pediatric TB and to enable concurrent detection of Mycobacterium tuberculosis (M. tuberculosis) and multi-drug-resistant tuberculosis (MDR) (38).

\subsection{Conclusions}

In the current study, the most common age for TB was the adolescent period (15 - 18 years). Adolescents and children with latent tuberculosis infection characterize the future reservoir of tuberculosis patients. Early detection and detailed assessment of adolescents at risk of developing infection are essential constituents of TB control. Further researches are required on the effect of vitamin D deficiency on responding immunity to TB diseases and the effect of iron deficiency on increasing severe form and mortality in pediatric patients with TB in Iran. Novel diagnostic tools, vaccine and programs are required in Iran for early detection and treatment of patients to achieve the goal of considerably decreasing the TB incidence by 2050.

\section{References}

1. World Health Organization . Global tuberculosis report 20152016. Available from: http://www.who.int/tb/publications/global_report/ gtbr2015.

2. WHO . Global Tuberculosis Report 2016. Available from: www.who. int/tb/publications/global_report/en/.

3. Mandalakas AM, Starke JR. Current concepts of childhood tuberculosis. Semin Pediatr Infect Dis. 2005;16(2):93-104. [PubMed:15825140].

4. Donald PR, Maher D, Qazi S. A research agenda to promote the management of childhood tuberculosis within national tuberculosis programmes. Int JTuberc Lung Dis. 2007;11(4):370-80. [PubMed: 17394681].

5. Zar HJ, Hanslo D, Apolles P, Swingler G, Hussey G. Induced sputum versus gastric lavage for microbiological confirmation of pulmonary tuberculosis in infants and young children: a prospective study. Lancet. 2005;365(9454):130-4. doi: 10.1016/S0140-6736(05)17702-2. [PubMed: 15639294].

6. Walls T, Shingadia D. Global epidemiology of paediatric tuberculosis. J Infect. 2004;48(1):13-22.

7. Shingadia D, Novelli V. Diagnosis and treatment of tuberculosis in children. Lancet Infect Dis. 2003;3(10):624-32.

8. WHO . Treatment of Tuberculosis: Guidelines for National Programmes. ; 2003.

9. United Nations Development Programme . The Prevention and Control of Tuberculosis in Iran 2011. Available from: www.undp.org.ir/ index.php/project-highlight/479-30-january.
10. World Health Organization . Global tuberculosis control 2010 2013. Available from: www.who.int/tb/publications/global_report/ 2010,Access.

11. Ghadiri K, Najafi F, Solimani B, Rezaei M, Afsharian M, Akramipour R. Childhood Tuberculosis in Kermanshah, Iran, During 10 Years. Arch Pediatric Infect Dis. 2013;1(3):131-5.

12. Sharifi-Mood B, Khalili M, Metanat M, Salehi M. Prevalence of Extrapulmonary Tuberculosis in Children, Zahedan, Iran. J Med Sci. 2006;6(1):52-4.

13. Phongsamart W, Kitai I, Gardam M, Wang J, Khan K. A population-based study of tuberculosis in children and adolescents in Ontario. Pediatr Infect Dis J. 2009;28(5):416-9. doi: 10.1097/INF.ob013e3181920d4d. [PubMed: 19352212].

14. Nejat S, Buxbaum C, Eriksson M, Pergert M, Bennet R. Pediatric tuberculosis in Stockholm: a mirror to the world. Pediatr Infect Dis J. 2012;31(3):224-7. doi: 10.1097/INF.0b013e31823d923c. [PubMed: 22094631].

15. Velayati AA, Mohammadi M. Childhood respiratory tuberculosis in Iran. Med J Islamic Republic Iran. 1990;4(1):1-4.

16. Cruz AT, Starke JR. Clinical manifestations of tuberculosis in children. Paediatr Respir Rev. 2007;8(2):107-17. doi: 10.1016/j.prrv.2007.04.008. [PubMed: 17574154].

17. Weber HC, Beyers N, Gie RP, Schaaf HS, Fish T, Donald PR. The clinical and radiological features of tuberculosis in adolescents. Ann Trop Paediatr. 2000;20(1):5-10. [PubMed: 10824206].

18. Kam A, Ford-Jones L, Malloy P, Khan K, Kitai I. Active tuberculosis among adolescents in toronto, Canada: clinical features and delays in diagnosis. Pediatr Infect Dis J. 2007;26(4):355-6. doi: 10.1097/01.inf.0000258700.86040.b6. [PubMed:17414403].

19. de Pontual L, Balu L, Ovetchkine P, Maury-Tisseron B, Lachassinne E, Cruaud P, et al. Tuberculosis in adolescents: A French retrospective study of 52 cases. Pediatr Infect Dis J. 2006;25(10):930-2. doi: 10.1097/01.inf.0000237919.53123.f4. [PubMed: 17006289].

20. Wu XR, Yin QQ, Jiao AX, Xu BP, Sun L, Jiao WW, et al. Pediatric tuberculosis at Beijing Children's Hospital: 2002-2010. Pediatrics. 2012;130(6):e1433-40. doi:10.1542/peds.2011-3742. [PubMed: 23184116].

21. UMDNJ . Management of latent tuberculosis in children and adolescent 2009. Available from: http://www.umdnj.edu/ntbcweb/ downloads/products/PediatricGuidelines.pdf.

22. Holmes $\mathrm{CB}$, Hausler $\mathrm{H}$, Nunn P. A review of sex differences in the epidemiology of tuberculosis. Int J Tuberc Lung Dis. 1998;2(2):96-104. [PubMed: 9562118].

23. Weiss MG, Sommerfeld J, Uplekar MW. Social and cultural dimensions of gender and tuberculosis. Int J Tuberc Lung Dis. 2008;12(7):829-30. [PubMed: 18544212].

24. Bouman A, Heineman MJ, Faas MM. Sex hormones and the immune response in humans. Hum Reprod Update. 2005;11(4):411-23. doi: 10.1093/humupd/dmio08. [PubMed: 15817524].

25. Marriott I, Huet-Hudson YM. Sexual dimorphism in innate immune responses to infectious organisms. Immunol Res. 2006;34(3):177-92. doi: 10.1385/IR:34:3:177. [PubMed: 16891670].

26. Fabri M, Stenger S, Shin DM, Yuk JM, Liu PT, Realegeno S, et al. Vitamin $\mathrm{D}$ is required for IFN-gamma-mediated antimicrobial activity of human macrophages. Sci Transl Med. 2011;3(104):104ra102. doi:10.1126/scitranslmed.3003045. [PubMed: 21998409].

27. Moussavi M, Heidarpour R, Aminorroaya A, Pournaghshband $Z$, Amini M. Prevalence of vitamin D deficiency in Isfahani high school students in 2004. Horm Res. 2005;64(3):144-8. doi: 10.1159/000088588. [PubMed: 16192739].

28. Rabbani A, Alavian SM, Motlagh ME, Ashtiani MT, Ardalan G, Salavati A, et al. Vitamin D insufficiency among children and adolescents living in Tehran, Iran. J Trop Pediatr. 2009;55(3):189-91. doi: 10.1093/tropej/fmn078. [PubMed: 18775944].

29. Dahifar H, Faraji A, Ghorbani A, Yassobi S. Impact of dietary and lifestyle on vitamin D in healthy student girls aged 11-15 years. J Med Invest. 2006;53(3-4):204-8. [PubMed:16953055]. 
30. Oppenheimer SJ. Iron and its relation to immunity and infectious disease. J Nutr. 2001;131(2S-2):616S-33S. [PubMed: 11160594] discussion 633S-635S.

31. Dallman PR. Iron deficiency and the immune response. Am J Clin Nutr. 1987;46(2):329-34. [PubMed: 3303900].

32. World Health Organization . Iron Deficiency Anemia Assessment ,Prevention, and Control 2001. Available from: www.who.int/nutrition/.../en/www.who.int/nutrition.

33. DeMaeyer E, Adiels-Tegman M. The prevalence of anaemia in the world. World Health Stat Q. 1985;38(3):302-16. [PubMed: 3878044].

34. Isanaka S, Mugusi F, Urassa W, Willett WC, Bosch RJ, Villamor E, et al. Iron deficiency and anemia predict mortality in patients with tuberculosis. J Nutr. 2012;142(2):350-7. doi: 10.3945/jn.111.144287. [PubMed: 22190024].

35. Schaaf HS, Marais BJ, Hesseling AC, Brittle W, Donald PR. Surveillance of antituberculosis drug resistance among children from the Western Cape Province of South Africa-an upward trend. Am J Public
Health. 2009;99(8):1486-90. doi: 10.2105/AJPH.2008.143271. [PubMed: 19197080].

36. Graham SM, Ahmed T, Amanullah F, Browning R, Cardenas V, Casenghi $\mathrm{M}$, et al. Evaluation of tuberculosis diagnostics in children: 1. Proposed clinical case definitions for classification of intrathoracic tuberculosis disease. Consensus from an expert panel. J Infect Dis. 2012;205 Suppl 2:S199-208. doi: 10.1093/infdis/jis008. [PubMed: 22448023].

37. Kaufmann SH, Hussey G, Lambert PH. New vaccines for tuberculosis. Lancet. 2010;375(9731):2110-9. doi: 10.1016/S0140-6736(10)60393-5. [PubMed: 20488515].

38. Nicol MP, Workman L, Isaacs W, Munro J, Black F, Eley B, et al. Accuracy of the Xpert MTB/RIF test for the diagnosis of pulmonary tuberculosis in children admitted to hospital in Cape Town, South Africa: a descriptive study. Lancet Infect Dis. 2011;11(11):819-24. doi:10.1016/S14733099(11)70167-0. [PubMed: 21764384]. 